\title{
Gam-COVID-Vac (Sputnik V): a heterologous adenoviral vector-based COVID-19 vaccine
}

\author{
Sanny Zi Lung Choo ${ }^{\mathrm{a}}$, Shyh Poh Teo ${ }^{\mathrm{a}, *}$ \\ ${ }^{a}$ Geriatrics and Palliative Unit, Department of Internal Medicine, Raja Isteri Pengiran Anak Saleha (RIPAS) Hospital, Ja- \\ Ian Putera Al-Muhtadee Billah, Bandar Seri Begawan BA1710, Brunei Darussalam.
}

\begin{abstract}
The Sputnik V COVID-19 vaccine (Gam-COVID-Vac) is an adenoviral vector-based vaccine against the SARSCoV-2 virus developed by Gamaleya National Research Centre for Epidemiology and Microbiology in Moscow, Russia. It consists of two recombinant adenovirus vectors; type 26 (rAd26) and type 5 (rAd5), with a heterologous approach used to reduce the risk of immune responses against the vector component. Findings from published studies showed that Gam-COVID-Vac was relatively safe, with mostly mild local and systemic reactions reported. Both rAd26-S and rAd5-S had to be administered to develop effective neutralising antibodies. No cross-reactive neutralising antibodies against the viral vector component was identified. The phase 3 trial performed in Russia only demonstrated an efficacy of 91.6\% in preventing COVID-19 disease. Common adverse events were flu-like symptoms, injection site reactions, headaches and asthenia, of which the majority were mild. Results from ongoing studies using Gam-COVID-Vac in Belarus, United Arab Emirates and India would be useful to demonstrate generalisability to other population groups. Details of adverse events and ongoing safety surveillance data are awaited before submission for regulatory approval.
\end{abstract}

Keywords: Adenovirus Vaccines, COVID-19, Russia, vaccines

The coronavirus disease 2019 (COVID-19) pandemic has caused significant loss of life globally. Older people are at high risk of developing infections and complications from the severe acute respiratory syndrome coronavirus 2 (SARS-CoV-2). Therefore, older people should be prioritized to receive COVID-19 vaccines [1]. As vaccines are being developed, there is an emerging body of evidence for the different vaccine platforms, which requires review in terms of vaccine safety and efficacy. This review describes the current evidence available for the Sputnik V COVID-19 vaccine (Gam-COVID-Vac).

Gam-COVID-Vac is a novel, heterologous adenoviral vector-based vaccine against the SARS-CoV-2 virus. It was developed by Gamaleya National Research Centre for Epidemiology and Microbiology in Moscow, Russia. This vaccine consists of two recombinant adenovirus vectors;

\footnotetext{
* Corresponding author: Shyh Poh Teo

Mailing address: Geriatrics and Palliative Unit, Department of Internal Medicine, Raja Isteri Pengiran Anak Saleha (RIPAS) Hospital, Jalan Putera Al-Muhtadee Billah, Bandar Seri Begawan BA1710, Brunei Darussalam.

Email: shyhpoh.teo@moh.gov.bn

Received: 20 April 2021 / Accepted: 23 June 2021
}

type 26 (rAd26) and type 5 (rAd5). Both vectors carry the gene for the full-length SARS-CoV-2 spike glycoprotein (rAD26-S and rAd5-S respectively). The vaccine is manufactured as two different formulations; the frozen GamCOVID-Vac $(0.5 \mathrm{~mL}$ per dose) and the lyophilized GamCOVID-Vac-Lyo, which has to be reconstituted in $1.0 \mathrm{~mL}$ sterile water for injection. The frozen vaccine is stored at $-18{ }^{\circ} \mathrm{C}$ and produced for large-scale use, while the lyophilized can be kept at 2 to $8{ }^{\circ} \mathrm{C}$, allowing delivery to remote areas of Russia [2].

The recombinant viral vector vaccine platform has been shown to induce antibody and cell-mediated immune responses, with known long-term safety data [3]. Other COVID-19 vaccines utilizing recombinant adenoviruses include Oxford-AstraZeneca (AZD1222), using a chimpanzee adenovirus (ChAdOx) [4], Janssen using Ad26 alone [5] and CanSino Biologics - Beijing Institute of Biotechnology using Ad5 alone [6].

Heterologous vaccination with two different viral vectors can be advantageous because the immune system may respond to the vector component in addition to the target antigen. This immune response to the vector component may result in reduced vaccine effectiveness, particularly if two doses are required. Therefore, the use of different viral vectors should theoretically overcome this problem and improve vaccine efficacy. This heterologous vaccina- 
tion approach was shown to be effective when used for the Ebola virus disease vaccine developed in Russia [7]. Thus, it was anticipated that Gam-COVID-Vac would be an effective vaccine to prevent COVID-19 disease.

In phase 1, participants received an intramuscular dose of rAd26-S or rAd5-S in the deltoid. For phase 2, a dose of rAd26-S was administered, followed by rAd5-S on day 21. Gam-COVID-Vac was used at Burdenko Hospital, while Gam-COVID-Vac-Lyo was administered at Sechenov University. At each locality, 38 volunteers were enrolled; 9 received $\mathrm{rAd} 26-\mathrm{S}$ and 9 received rAd5-s (Phase 1); while 20 received rAd26-S followed by rAD5-S on day 21 (Phase 2). The majority of the participants were men in the late 20 s or early 30 s [2].

In terms of safety data, most local and systemic reactions were mild. No serious adverse events were reported. The most common reactions were localized pain at the injection site, fever, headache, fatigue and myalgia; with more adverse events occurring after the second dose. In the phase 1 studies, the SARS-CoV-2 receptor binding domain (RBD) specific IgG was detected on day 14 in $88.9 \%$ after rAd26-S, and $84.2 \%$ after rAd5-S. This immune response increased to $100 \%$ on day 21 . However, both rAd26-S and rAd5-S had to be administered to develop neutralizing antibodies at day 42 in $100 \%$ of participants. Participants receiving rAd26-S alone had a seroconversion rate of $61.1 \%$ at day 42.

For the phase 2 studies, post-vaccination ELISA titers were significantly higher than convalescent plasma post COVID-19 infection at day 28 and 42. Cell-mediated responses were also detected in all vaccine recipients by day 28 . There were no cross-reactive neutralizing antibodies against the viral vector component. This was demonstrated using the serum of participants on day 28 after immunization, where neutralizing antibodies to $\mathrm{rAd} 26 \mathrm{did}$ not cross-react with rAd5 [2].

The phase 3 trial was carried out in Russia only, with 21 977 adults aged above 18 enrolled in the randomized, double-blind, placebo-controlled study at 25 centers across Moscow. Participants were randomly assigned (3:1) to receive Gam-COVID-Vac vaccine or placebo. The vaccine group received $\mathrm{rAd} 26$ and $\mathrm{rAd} 5$ vector-based heterologous prime-boost COVID-19 vaccine at least 21 days apart while the placebo group received the vaccine buffer composition. The efficacy rate was $91.6 \%$ from 21 days after the first dose (the day of the second dose), with 16 of 14964 vaccine recipients and 62 of 4902 placebo recipients contracting COVID-19 disease. This efficacy was consistent across all age groups, with the oldest group being above 60 years old.

The most common adverse events reported were flu-like symptoms, injection site reactions, headaches and asthenia. The majority (94\%) of reported adverse events were mild or Grade 1 symptoms [8].

There were certain limitations with this trial. As the study was performed in Moscow with the majority (98.5\%) of participants being white, it remains to be seen if these results could be extrapolated to other population groups.
Results from the ongoing clinical trials using Gam-COVID-Vac in Belarus, United Arab Emirates and India would be useful to assess generalisability. The phase 3 trial was only performed with the frozen preparation stored at -18 ${ }^{\circ} \mathrm{C}$, with the assumption that the lyophilized formulation provides the same efficacy. The latter has been approved for use by the Ministry of Health of the Russian Federation for practical ease of global distribution.

There may an under-diagnosis of COVID-19 cases, as asymptomatic PCR-confirmed COVID-19 cases were not included in the efficacy analyses. However, similar to other trials, the main outcome was symptomatic COVID-19 disease. Thus no PCR-testing was done beyond 21 days of the first dose unless participants developed symptoms suggestive of COVID-19. It would also be useful to have efficacy data on day 21 and 42 of the trial. This would allow time for an immunologic response to develop after the first and second dose. In terms of safety data, only a preliminary report of adverse events following immunization was provided. There were 68 participants who experienced 70 episodes of serious adverse events among more than 21000 participants, of whom more than 16000 received the vaccine. The authors stated that further details would be made available in a future publication regarding these adverse events. This additional information would be required before submission for regulatory approval.

The main findings from the Phase 3 trial for Gam-COVID-Vac relevant to older people were as follows: There was a similar efficacy in younger and older people. This was reassuring, given the theoretical risk of a reduced vaccine response in older people due to immune senescence [1]. In terms of adverse events, while the data available was not age-stratified, there was only a small number of serious adverse events identified at the time of interim analysis.

For COVID-19 vaccines, the Phase 3 studies should demonstrate the effectiveness and that benefits outweigh the risks to obtain Emergency Use Authorisation (EUA) from regulatory authorities. Monitoring of efficacy and safety data usually requires a 2-month median follow-up period after completion of the full vaccination regimen. For example, as natural immunity to coronavirus infections is usually short-lived, the significant waning of protective responses may become noticeable in the second month [9]. After Phase 3 trials, researchers should also strive to optimize vaccine regimens, including dosing intervals for booster doses. Fractional dosing or lower doses of vaccine with maintained efficacy may allow more people to be vaccinated. Specific patient groups, such as pregnant women and children should also be evaluated. Longer term follow-up is required to assess the durability of protective immune responses and surveillance for adverse events after immunization. Consideration should also be given to effective coordination of post-efficacy trial tasks, such as mass production while maintaining the quality of vaccines; and coordinating supplies and distribution. Scaled-up production may cause problems with the vector, purification process or formulation, which needs to meet 
international quality standards before use [10].

Finally, there should be ongoing evaluation of vaccine efficacy against emerging new SARS-CoV-2 variants. The variants 501Y.V1 (B.1.1.7) in the United Kingdom and 501Y.V2 (B.1.351) in South Africa have the mutation N501Y in the receptor-binding domain of the spike protein, contributing to increased transmission. However, for the 501Y.V2 variant, two additional mutations (E484K and $\mathrm{K} 417 \mathrm{M}$ ) in the spike protein provide a potential immune escape from antibodies. Studies have suggested that there is reduced efficacy of current vaccines, particularly against the South Africa variant [11]. Thus, the production of COVID-19 vaccines should be reactive and adapt to these newly emerging lineages to remain effective against the main circulating variants.

In conclusion, the Phase 3 studies showed early promising evidence that Gam-COVID-Vac was efficacious in preventing SARS-CoV-2 infections, including in older people. Certain limitations in the studies should be taken into account, with a need for more specific data on older people and emerging SARS-CoV-2 variants. Post-marketing surveillance for this vaccine is also required for ongoing monitoring of adverse events following vaccination.

\section{Declaration}

Conflict of interest: The author declared no conflict of interest.

\section{References}

1. Jang J M, Choi H S, Lee J S, et al. Femoral intertrochanteric fractures of the patients in the emergency department due to minor falls: special consideration in the middleold to oldest-old patients. Annals of geriatric medicine and research, 2019, 23(3): 125.

2. Logunov D Y, Dolzhikova I V, Zubkova O V, et al. Safety and immunogenicity of an rAd26 and rAd5 vector-based heterologous prime-boost COVID-19 vaccine in two formulations: two open, non-randomised phase $1 / 2$ studies from Russia. The Lancet, 2020, 396(10255): 887-897.

3. Rauch S, Jasny E, Schmidt K E, et al. New vaccine technologies to combat outbreak situations. Frontiers in immunology, 2018, 9: 1963.

4. Chan J F W, Yuan S, Kok K H, et al. A familial cluster of pneumonia associated with the 2019 novel coronavirus indicating person-to-person transmission: a study of a family cluster. The lancet, 2020, 395(10223): 514-523.

5. Tegally H, Wilkinson E, Giovanetti M, et al. Emergence and rapid spread of a new severe acute respiratory syndrome-related coronavirus 2 (SARS-CoV-2) lineage with multiple spike mutations in South Africa. medRxiv, 2020.

6. Zhu F C, Guan X H, Li Y H, et al. Immunogenicity and safety of a recombinant adenovirus type-5-vectored COVID-19 vaccine in healthy adults aged 18 years or older: a randomised, double-blind, placebo-controlled, phase 2 trial. The Lancet, 2020, 396(10249): 479-488.

7. Dolzhikova I V, Zubkova O V, Tukhvatulin A I, et al. Safety and immunogenicity of GamEvac-Combi, a heterologous VSV-and Ad5-vectored Ebola vaccine: an open phase I/ II trial in healthy adults in Russia. Human vaccines \& immunotherapeutics, 2017, 13(3): 613-620.

8. Logunov D Y, Dolzhikova I V, Shcheblyakov D V, et al. Safety and efficacy of an rAd26 and rAd5 vector-based heterologous prime-boost COVID-19 vaccine: an interim analysis of a randomised controlled phase 3 trial in Russia. The Lancet, 2021, 397(10275): 671-681.

9. Krause P R, Gruber M F. Emergency use authorization of Covid vaccines-safety and efficacy follow-up considerations. New England Journal of Medicine, 2020, 383(19): e107.

10. Kim J H, Marks F, Clemens J D. Looking beyond COVID-19 vaccine phase 3 trials. Nature medicine, 2021, 27(2): 205-211.

11. Fontanet A, Autran B, Lina B, et al. SARS-CoV-2 variants and ending the COVID-19 pandemic. The Lancet, 2021, 397(10278): 952-954. 